\title{
COMHUMANANITAS
}

Revista ComHumanitas, ISSN: 1390-776X

Vol. 11, núm. 3 (2020), Septiembre - Diciembre 2020 DOI: https://doi.org/10.31207/rch.v11i3.276

\section{Verificación de hechos: Falibilismo de Popper y la razonabilidad de las masas}

\author{
Fact Check: Popper's Fallibilism and the \\ reasonableness of the masses
}

\section{Verificação de fatos: falibilismo de Popper e a razoabilidade das massas}

\author{
Gabriela Lourdes Vélez Bermello \\ Universidad Estatal Península de Santa Elena (Ecuador) \\ gvelezb@upse.edu.ec \\ Wilson Benjamín León Valle ${ }^{2}$ \\ Universidad Estatal Península de Santa Elena (Ecuador) \\ wleon@upse.edu.ec
}

Fecha de recepción: 3 de noviembre de 2020

Fecha de recepción evaluador: 15 de noviembre de 2020

Fecha de recepción corrección: 20 de diciembre de 2020

\footnotetext{
${ }^{1}$ Gabriela Lourdes Vélez Bermello, licenciada en Ciencias de la Comunicación y magister en Periodismo. Estudiante doctoral en la Universidad Nacional de Rosario - Argentina. Laboró como docente de la Facultad Ciencias de la Comunicación de la Universidad Laica Eloy Alfaro de Manabí y actualmente es docente de la Universidad Estatal Península de Santa Elena (UPSE). Docente investigadora. Además, trabajó en medios de comunicación EDIASA, en medios radiales como: Gaviota, Son de Manta y Radio Visión. Fue directora del Departamento de Comunicación del Ministerio del Interior para Manabí. Profesional convergente, con más de 14 años de experiencia en redacción periodística, dirección de medios, fotografía, maquetación, sonde de medios, asesoría, factcheking y contenidos digitales. ORCID: https://orcid.org/0000-0002-7414-8441

2 Wilson León Valle, Teólogo, misionero, filósofo, académico, comunicador, educador, activista de los Derechos Humanos, proyectista y experto en temas de desarrollo endógeno. Docente investigador de la Universidad Estatal Península de Santa Elena (UPSE). ORCID: https://orcid.org/0000-0002-3374-5207
} 


\title{
Resumen
}

La verificación de información es parte del ejercicio periodístico, esta puede pasar por un proceso de falibilismo, sin embargo, muchos factores restan credibilidad a la profesión. El objetivo del artículo es identificar cómo la ciudadanía, en especial los futuros comunicadores, asumen la labor del ejercicio periodístico, con respecto a sus rutinas productivas, si este le brinda tratamiento al hecho noticioso, y de qué manera las personas procesan la información. Es importante destacar cómo los sujetos disciernen los contenidos noticiosos. El trabajo presenta un enfoque mixto de investigación; cualicuantitativo, la población objeto de estudio está conformada por estudiantes pertenecientes a dos universidades del Ecuador. Se aplicó el tipo de muestreo no probabilístico y entre las conclusiones se pueden desatacar que la credibilidad de la actividad, desde sus preceptos éticos y de responsabilidad social, está en tela de juicio. Además, el entorno digital actual está distorsionando las funciones sustantivas que conlleva la profesión, mientras tanto, la ciudadanía está optando por consumir contenidos sin profundizar en un pensamiento crítico coherente. Existe un incremento exponencial de noticias falsas y poca contrastación de información y, finalmente, la inmediatez dentro de los medios de comunicación está superando a la confirmación de los hechos que se publican. Los resultados denotan un futuro poco alentador en el entorno de esta labor, pero que con nuevos replanteamientos podrían cambiar.

Palabras clave: Comunicación; Ecuador; rutinas productivas; ética; factcheking; fake news.

\begin{abstract}
The verification of information is part of the journalistic exercise, it can go through a process of fallibilism, however, many factors reduce the credibility of the profession. The objective of the article is to identify how citizens, especially future communicators, assume the work of journalism, with respect to their productive routines, if it treats the news event, and in what way people process the information. It is important to highlight how the subjects discern the news content. The work presents a mixed research approach; qualitative-quantitative, the study population is made up of students belonging to two universities in Ecuador. The type of non-probabilistic sampling was applied and among the conclusions it can be unleashed that the credibility of the activity, from its ethical and social responsibility precepts, is in question. In addition, the current digital environment is distorting the substantive functions that the profession entails, meanwhile, citizens are choosing to consume content without delving into coherent critical thinking. There is an exponential increase in false news and little information contrast and, finally, the immediate one within the media is exceeding the confirmation of the facts that are published. The results indicate a bleak future in the environment of this work, but with new rethinking could change.
\end{abstract}

Keywords: Communication; Ecuador; productive routines; ethics; factchecking; fake news. 


\section{Resumo}

A verificação de informações faz parte do exercício jornalístico, pode passar por um processo de falibilismo, porém muitos fatores reduzem a credibilidade da profissão. $\mathrm{O}$ objetivo do artigo é identificar como os cidadãos, especialmente os futuros comunicadores, assumem o trabalho jornalístico, no que diz respeito às suas rotinas produtivas, se trata o acontecimento noticioso e de que forma as pessoas processam a informação. É importante destacar como os sujeitos discernem o conteúdo das notícias. O trabalho apresenta uma abordagem de pesquisa mista; Quali-quantitativo, a população estudada é formada por estudantes de duas universidades do Equador. Foi aplicado o tipo de amostragem não probabilística e dentre as conclusões pode-se destacar que a credibilidade da atividade, a partir de seus preceitos éticos e de responsabilidade social, está em questão. Além disso, o ambiente digital atual está distorcendo as funções substantivas que a profissão acarreta, enquanto os cidadãos estão optando por consumir conteúdo sem mergulhar em um pensamento crítico coerente. Há um aumento exponencial de notícias falsas e pouco contraste de informações e, por fim, o imediatismo dentro da mídia está excedendo a confirmação dos fatos publicados. Os resultados indicam um futuro sombrio no ambiente deste trabalho, mas com novo repensar pode mudar.

Palavras-chave: Comunicação; Equador; rotinas produtivas; ética; verificando os fatos; notícias falsas.

\section{Introducción}

A lo largo de la historia periodística se ha entendido a este ejercicio profesional como un medio para transmitir información que debe estar previamente verificada y contrastada. De hecho, muchos textos relacionados a las rutinas productivas detallan cómo son las jornadas de recolección de datos, empezando por reuniones matutinas con los editores, coberturas, tratamiento de la noticia y publicación sea el formato que fuere.

Sin embargo, en los últimos años ha tomado fuerza aquello que se conoce como verificación de hechos o factchecking; se trata de organizaciones creadas para comprobar si lo que un medio de comunicación difunde es verdadero o falso e incluso hay portales webs creados con este fin, los cuales no sólo califican de verdadero o falso, sino que colocan otras etiquetas como, insostenible, dudoso, sí, pero, entre otros.

Laura Zommer (2014), una de las precursoras del factchecking en América Latina, expone que el método por el cual se realiza una verificación del discurso posee 4 etapas y 8 pasos. El primer paso dentro de la primera etapa es: seleccionar una frase del ámbito público que esté difundida en algún medio de comunicación o que sea parte de un discurso político, este paso se lo hace tomando en cuenta que cuestiones relacionadas con la religión, farándula, deportes y otros, no son objeto de chequeo. 
En lo mencionado, pudiera existir un sesgo por la elección subjetiva de la frase, pero luego, en el segundo paso de la primera etapa se: pondera su relevancia y es aquí en el que Zommer explica, "este es el paso del método en el que el periodista y su editor tienen un rol protagónico y donde se pone en juego el criterio periodístico" (Zommer, 2014), entonces la elección a discreción de la frase por su relevancia podría estar calando en una mera subjetividad.

En este sentido se plantean las siguientes preguntas: ¿cómo se está seguro de que dicha frase es la que el lector necesita conocer si es verdad o no? y ¿cómo se garantiza que la selección de la frase no esté ligada a intereses del equipo de chequeo o de la misma organización? Además, es necesario sostener que la verdad tiene sus diferentes dimensiones, la misma puede ser una adecuación del discurso a la realidad, enlazado entre la coherencia de lo que se sabe y se piensa e incluso desde la verdad más allá de los hechos, temas que están sustentados bajos los análisis de Verón (2002) y Todorov (1972).

Las siguientes etapas y pasos del factchecking están enlazadas a las consultas de fuentes, contextualizar y calificar, este sistema que sería una especie de rutina productiva se ve justificado por la cantidad de Fake News que han existido y existen hasta la actualidad, mucho más potencializados con Internet.

Esta acción ha sido aprobada por autores quienes deliberan que, con la gran demanda de información falsa, era necesario contar con organizaciones encargadas de catalogar lo que es real, pero otros investigadores consideran que la verificación de información era ya una práctica sine qua non dentro de las rutinas periodísticas. Es decir, todo medio de comunicación desde su interior ejecuta esta práctica de verificación de datos o al menos en teoría es lo que se piensa.

En este ámbito periodístico, la parte medular es el proceso que se le da a la generación de contenidos dentro de las salas de redacción, las que han dejado cierta desconfianza en la profesión cayendo en sesgos, noticias falsas, en copyright, como dice Smiers (2006) y prejuicios a la hora de informar, “¿por qué se repite tanto esta mala práctica periodística? Porque en las salas de redacción hace falta la pausa en medio del vértigo", afirma Buitrón (2005).

Es oportuno recordar lo que afirman autores como Pascual (2016) que para obtener un buen producto es considerable que todas las noticias sean verificadas, donde el comunicador esquive sus opiniones, sentimientos e intereses.

Con esta antesala y poniendo en evidencia el accionar periodístico actual desde el factchecking este texto se enfoca hacia lo epistemológico, concebido por GonzálezSaibene (2011) como "el acto político del ejercicio de la crítica". Además, se esbozarán y alanzarán tres aristas filosóficas:

1.- Las categorías filosóficas que envuelven al factchecking.

2.- La verdad como adecuación en el ejercicio periodístico, arraigadas en el falibilismo (falsación infinita) de Popper. 
3.- La razonabilidad de las masas dependientes de su propio conocimiento del entorno.

\section{Marco Referencial}

El cuestionamiento de la práctica periodística podría frenarse consolidando las bases filosóficas, por ello, es preciso indicar que esta profesión trabaja en referencia a la realidad, opinión, ética, verdad, objetividad, hechos, etcétera. (Pujante, 2012, p. 895). Quizás estas categorías filosóficas son llevadas a cabo consciente o inconscientemente por los comunicadores e incluso con conceptos desdibujados.

Si se comienza a profundizar en la pregunta que el filósofo Watzlawick (2011) hace sobre ¿hasta qué punto es real lo que ingenuamente y sin el menor reparo solemos llamar realidad?, se llegaría a un debate que se ha marcado a lo largo de la historia.

Y, probablemente un filósofo que se refería a la construcción de la realidad fue Platón desde su idealismo, quien, al exponer el Mito de la Caverna, brindó un claro ejemplo de las construcciones mentales que pueden tener las personas, donde las sombras de la realidad casi nunca o nunca son la realidad en sí misma.

De igual forma, la mirada que tienen los medios de comunicación estaría intoxicada y sesgada por la mirada del periodista. Entonces, la postura de la realidad que ofrecen los medios de comunicación se ve perturbada por las diversas apreciaciones de la gente que hace periodismo. De hecho, autores como Nietzsche o Heidegger reafirman lo dicho.

Este sistema mediático, instaurado por las grandes esferas del poder, ya está pensado para y por el consumidor de información. Pero ¿qué sucede en las personas con la información que recibe de los medios de comunicación?, es muy probable que la información conviva de una verdad como adecuación. Esto porque el periodismo aún se mantiene de lo que se determina como objetivad o probablemente falsa objetividad en sus procedimientos, haciendo de su trabajo una búsqueda por datos corroborados.

Aunque se piense que el periodista está pendiente de la coincidencia de los hechos con su entorno real, aquello que ejerce no es ciencia como tal, sino modestamente una investigación o intervención social en la que sí pueden citarse ciertos datos que nacen de la ciencia. "Sólo hay una teoría de la verdad que pueda ser realmente tomada en serio: la teoría de la verdad como adecuación, la teoría que afirma que un enunciado es verdadero si coincide con los hechos, con la realidad”. (Popper, 1998:28).

De hecho, Popper veía a la televisión como una amenaza para la libertad (Popper y Candri, 1994).

Por ejemplo, el factchecking trabaja en la búsqueda de datos que bien pueden tener su génesis de investigaciones científicas o en el procesamiento de big data, con 
grandes volúmenes de información, no obstante, eso es un parte de la miscelánea de datos entre el saber y la ciencia que compone un producto comunicacional.

Ese esfuerzo por llevar datos certeros en una información puede verse entorpecidos por múltiples factores, tomando en cuenta el falibilismo, nacido desde Charles Sanders Peirce, en la cual ningún conocimiento es absoluto porque todas las teorías son aproximativas y susceptibles de correcciones y de falsedad.

A esto, Popper menciona que una proposición es científica si puede ser refutable, es decir, susceptible de que en algún momento se puedan plantear ensayos o pruebas para refutarla independientemente de que salgan airosas o no de dichos ensayos. Todo esto pensado desde el racionalismo crítico.

En cierto modo, el periodismo, y en este caso el factchecking, enfrascado hacia cualquier método de descripción de lo que pasa en el planeta en la búsqueda por la verdad de los hechos, podría falsearse; el problema está en que esta profesión olvida lo que es neutral pensando que su accionar en coberturas es pura y limpia objetividad, pero la ciencia como tal no trabaja de esa forma. Quizás es necesario saber diferenciar entre lo que es objetivo y neutral (Habermas, 1989).

Incluso si se centra en la profesión, considerando que los periodistas trabajan bajo "normas éticas" y con una 'objetividad absoluta', bastará con encontrar a un solo comunicador que no se rija a ello para falsear esa aseveración que sigue manteniendo la profesión.

Ante esto Verón (1972) convoca al periodismo a desembarazarse de la pretensión de objetividad, que fue un paradigma del siglo XX y posiblemente el periodismo actual no lo ha digerido.

En el libro la Construcción del Acontecimiento, Verón (2002) detalla ese "sagrado principio de objetividad" que parece actuar como eje de la representación del mundo a la que dicen adherirse los medios de comunicación.

Los acontecimientos sociales no son objetos que se encuentran ya hechos en alguna parte en la realidad y cuyas propiedades y avatares nos son dados a conocer de inmediato por los medios con mayor o menor fidelidad. Sólo existen en la medida en que esos medios los elaboran. (Verón, 2002, p.2)

Entonces, el factchecking que nace del periodismo y es conformado en mayor grado por periodistas sería acaso una especie de realidad paralela.

Frente a aquello, se debería entender que el periodismo no pretende obtener una verdad absoluta, pero que el periodista debe intentar alcanzar los datos más limpios y verídicos, que pudieran surgir de la ciencia como tal.

Mientras tanto, los periodistas están limitados a recoger o recolectar información como algo verosímil o creíble. 
La experiencia organizativa del periodista le impone prejuicios en contra de las posibilidades contrarias a las expectativas preexistentes. Desde el punto de vista de un periodista, sin embargo, sus experiencias con otras organizaciones durante un período de tiempo validan sus juicios periodísticos y pueden reducirse al sentido común. Por «sentido común» el periodista entiende lo que la mayoría de los periodistas creen verdad o dan por sentado (TUCHMAN, 1998/1999, p. 2 - 11).

En consonancia con lo expuesto se concibe también que un periodista tendrá otros obstáculos para obtener información veraz, como:

- $\quad$ Cuando el periodista no estuvo en el momento exacto del hecho.

- Cuando la fuente no estaba en sus cabales (drogado, borracho, con problemas mentales y emocionales)

- $\quad$ Cuando las fuentes oficiales no cuenten con cifras correctas, etc.

- $\quad$ Cuando hay intereses que contaminan sus enunciados.

- $\quad$ Y/o por construcciones discursivas y su relación con el poder.

Pero esa búsqueda por la realidad puede verse afectados por temas éticos, normativos y deontológicos.

\subsection{La mirada del receptor / perceptor / prosumidor.}

Dejando de lado la visión del periodista y pasando al lado del consumidor de información: en una construcción de realidad el consumidor de la información creerá lo que desee creer de acuerdo con sus conocimientos del mundo en general, su nivel de estudio, cultura, lenguaje y desde las mismas representaciones colectivas que influyen en la codificación y decodificación de información. Todo esto frente a de lo que refiere Wittgenstein (1922) de una palabra para cada objeto en la totalidad de la realidad.

Habrá casos donde la propia gente acepte o deseche algo noticioso, por ejemplo:

- $\quad$ Cuando el consumidor de información sepa del tema que aborda la noticia y pueda discernir entre verdadero o no.

- $\quad$ Cuando el consumidor ilustrado busque otros medios para contrastar.

- $\quad$ Cuando el consumidor tenga a disposición base de datos que nazcan desde trabajos científicos.

- Cuando el consumidor de información tenga ya representaciones admitidas colectivamente.

- $\quad$ Y, sobre todo, cuando el consumidor de información desee corroborar dicha noticia.

Este último punto denota que no todas las personas están interesadas en corroborar tal o cual información, pues algunos prefieren quedarse con una sola verdad, por tal 
motivo, si se extrapola esto al factchecking se entendería que quienes visitan la página web de estas organizaciones o siguen sus redes sociales, es gente ilustrada o medianamente ilustrada que busca un trabajo periodístico más prolijo.

En otras palabras, como diría Alfred Schutz (1974, p. 121-124) estaríamos hablando de "el ciudadano bien informado" quien recurriría a las plataformas de factchecking, según la distribución social del conocimiento de tipos ideales al que se le añade: "el experto" y "el hombre de la calle". Esto en contra de lo que Vattimo (1994) afirma, pues para él quienes están inmersos en medios de comunicación, no llegan a ser parte de una comunidad ilustrada.

Entonces, el sujeto interpretará las informaciones simple y sencillamente a su modo, teniendo como premisa que la constitución del sujeto autónomo que es capaz de gobernar y gobernarse configurando nuevas instituciones que no quedan establecidas de una vez y para siempre, sino que van modificándose de acuerdo las configuraciones ciudadanas y de los gobiernos (Castoriadis, 2002).

Por otro lado, si desde los gestores del periodismo no hay una distinción entre conocimiento científico de lo no científico, se seguirá produciendo información que desinforma o se encajaría al periodismo en un conocimiento especulativo que no necesita verificación. En esa misma corriente, hay que entender que el lenguaje es construido por el sujeto, pero también construye sujetos.

La epistemología normativa deja en jaque al periodismo con ese constructo lógico e idealizado de práctica profesional que pretende ser el reflejo de la realidad y, además, sugiere cambiar el término verdad por veracidad en la acción informativa. Pues, como Luhmann (2000) expresa, la realidad para un periodista sería la selección o un extracto de un hecho porque lo que sucede en el planeta es mucho mayor que la realidad de un acontecimiento mediático.

Es fundamental deducir a partir de qué enfoque y concepto se mira a esta profesión y para esto debe estar monitoreado desde una vigilancia epistemológica (Bachelard, 1984), como disciplina social y por ende pragmática.

La oleada de noticias falsas y el escaso control haría que se replantee en un futuro la labor periodística porque, así como Bauman (2015) declaró que atraviesan tiempos de modernidad líquida, donde el consumismo impera, la gente está ávida de información rápida haciendo que la profesión no cuente con un enfoque epistemológico. Sin embargo, el usuario o consumidor de información está a la espera de la credibilidad de los datos vertidos en la información, por ello el requerimiento también de observar al periodismo desde la deontología.

Hay que concebir que una cuestión es la investigación periodística en la intervención mismo porque el objetivo no es la producción del conocimiento en general, sino que el periodismo se apoya en datos científicos encuadrados en esa situación, pues no le interesa la formulación teórica, le interesa la veracidad del hecho. 
Si desde el siglo XX se ha puesto bajo sospecha la actividad periodística con escuelas como las de Francfort, qué evitaría dudar de las prácticas de factchecking que cae en subjetividades desde la elección de la frase y ponderación de esta, tal como se cita en los primeros párrafos de este texto.

Parrilla (2019) manifiesta que el proceso y auge que ha tenido el chequeo de datos es sólo un mecanismo que brinda la sensación de seguridad al consumidor de noticias, sustituyendo el poder que tenían los medios de comunicación, pero no es la solución, sino quizás un espejismo y "si algún día el ser humano dispone de una herramienta que le permita escapar de sus sesgos y le muestre la realidad tal cual es, podemos tener algo por seguro: no la habrá desarrollado un periodista" (Parrilla 2019).

No obstante, hay otras formas epistemológicas para mirar al periodismo, como, por ejemplo, desde la lingüística con la parte metaepistemológica y en sí desde el interpretativismo, a partir de la filosofía del lenguaje pragmático como la abordada Wittgenstein, Peirce y Austin. También por otros autores como Eco (1992) desde la interpretación y sobreinterpretación, porque la lógica mediática va más allá de una comercialización social del conocimiento y del factchecking el cual, quizás no es el oasis en el desierto hacia la búsqueda de la Teoría de la Verdad.

Y, finalmente, siempre será bueno hacer una crítica desde la naturalización del ejercicio periodístico porque si la profesión no tiene sus bases bien establecidas el producto que genere será ambiguo.

La verificación de información, como parte del ejercicio periodístico puede pasar por un proceso de falibilismo, sin embargo, al ser la comunicación un proceso complejo y amplio, el factchecking se vuelve algo minúsculo frente a las masas, quienes actuarán acorde a sus pensamientos previos.

A propósito de la búsqueda incesante de datos que sirvan al periodista en la elaboración de productos comunicacionales, habría que pensar en desacralizar la ciencia y acercarla más hacia la sociedad.

"El periodismo y la comunicación científica pueden ser vistos como una pareja cuya unión y funciones complementarias son necesarias para producir una mejor comprensión pública de la ciencia..., ambos son vitales”. (Janowski, 2013)

\section{Metodología}

Para el desarrollo de la investigación se procedió a la revisión de los diferentes enfoques que se presentan en la metodología de la investigación, para optar por el más viables que fundamente y justifique el estudio en el territorio, En este contexto se propuso el enfoque mixto desde la perspectiva de Hernández Sampieri; con el fin de cualificar, pero también, cuantificar la actividad el ejercicio profesional desde una dualidad; periodismo - sociedad. En este sentido se pretende determinar de qué manera el periodismo le da el tratamiento al hecho noticioso, medios e instrumentos, cómo la procesa y cómo la asume la ciudadanía. 
La población objeto de estudio está conformada por los estudiantes pertenecientes a dos universidades de la zona marina costera; Universidad Estatal Península de Santa Elena (UPSE), carrera de Comunicación y la Universidad Laica Eloy Alfaro de Manabí (ULEAM); carrera de comunicación, periodismo y publicidad.

El tipo de muestreo que se aplicó es el muestreo por conveniencia, es el tipo de muestreo no probabilístico en el que los sujetos son seleccionados dada la conveniente accesibilidad y proximidad, se la usa también cuando la población es demasiado grande y resulta imposible incluir a cada individuo. También, este tipo de muestreo se aplica a estudiantes voluntarios como sujeto de investigación.

Para el procedimiento de la recolección de datos, se envió el instrumento a los estudiantes de las universidades, para que procedan a responder la encuesta de forma voluntaria, el número de estudiantes que respondieron la encuesta fue de 361 ; los mismos que corresponden a 259 estudiantes de la UPSE y de 101 de la ULEAM. El tipo de estudio por la temporalidad es transversal, esto es con el fin de estimar la magnitud de la problemática planteada en un momento dado. La tabulación y análisis de resultado se lo realizó con el SPSS Analysis Software.

\section{Hallazgos}

Da los 31 Ítems, que conforman el instrumento denominado encuesta, los autores han escogido, el proceso para la selección de las preguntas para presentar, esta acción resultó compleja, sin embargo, se lograron concretar algunas características de las respuestas que permiten fundamentar con mayor propiedad la investigación, estas se presentan a continuación:

Tabla 1. ¿Considera que los contenidos noticiosos están alineados a la subjetividad del periodista?

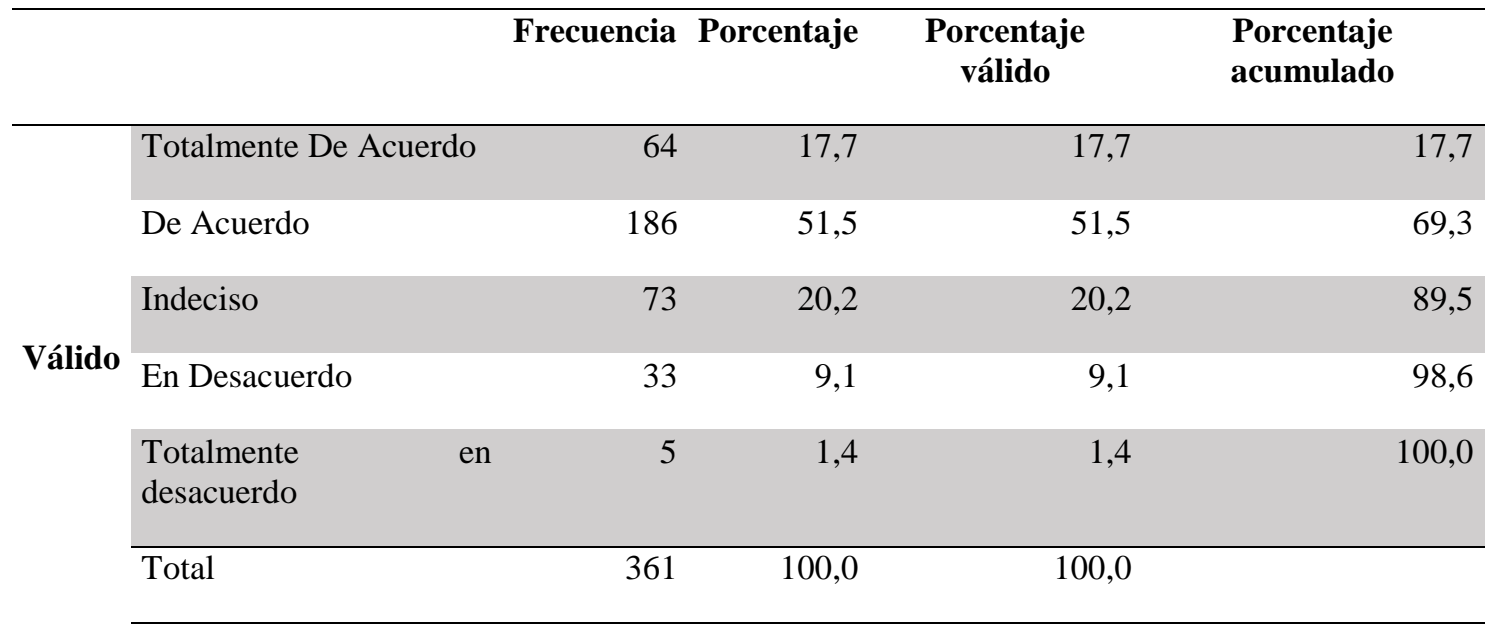

Fuente: Estudiantes universitarios de la ULEAM - UPSE. Elaborado por: autores

La respuesta a esta pregunta está directamente relacionada con la subjetividad que presenta el periodista a la hora de emitir los contenidos noticiosos, la respuesta de los encuestados indican que el $69,3 \%$ del porcentaje acumulado manifiesta que está 
totalmente de acuerdo y de acuerdo con del hecho en que los contenidos noticiosos están alineados a la subjetividad del periodista, interesante, porque la respuesta se puede entender como un llamado de atención con relación a la postura ética profesional de la actividad periodística.

Frente al 30,7\% entre personas indecisas y que se encuentran en totalmente en desacuerdo con la que se plantea en la pregunta.

Tabla 2. ¿Considera que los periodistas, en el ejercicio profesional, aplican el código deontológico y demás artículos sustentados en la Ley Orgánica de Comunicación?

\begin{tabular}{|c|c|c|c|c|c|}
\hline & & Frecuencia & Porcentaje & Porcentaje válido & Porcentaje acumulado \\
\hline & Siempre & 50 & 13,9 & 13,9 & 13,9 \\
\hline & Usualmente & 187 & 51,8 & 51,8 & 65,7 \\
\hline Válido & Ocasionalmente & 115 & 31,9 & 31,9 & 97,5 \\
\hline & Usualmente no & 9 & 2,5 & 2,5 & 100,0 \\
\hline & Total & 361 & 100,0 & 100,0 & \\
\hline
\end{tabular}

Fuente: Estudiantes universitarios de la ULEAM - UPSE. Elaborado por: autores.

La pregunta número dos concierne directamente al ejercicio profesional de los periodistas e indica si estos aplican el código deontológico en el desarrollo de la actividad, además de los preceptos manifestados en la Ley Orgánica de Comunicación (LOC). En cuanto a esta pregunta el 13,9\% indica que siempre lo que le resta credibilidad a la tarea el 51,8\% manifiesta que usualmente considera que los periodistas en su profesión aplican el código deontológico, un 31,9\% de ocasionalmente usan los instrumentos mencionados, finalmente un $2,5 \%$ que indica que usualmente no aplican el código deontológico y no sustentan su actividad en la LOC.

Tabla 3. ¿Los medios que producen información falsa lo hacen para manipular la opinión pública?

\begin{tabular}{|c|c|c|c|c|c|}
\hline & & Frecuencia & Porcentaje & Porcentaje válido & Porcentaje acumulado \\
\hline & Siempre & 171 & 47,4 & 47,4 & 47,4 \\
\hline & Usualmente & 143 & 39,6 & 39,6 & 87,0 \\
\hline \multirow[t]{3}{*}{ Válido } & Ocasionalmente & 39 & 10,8 & 10,8 & 97,8 \\
\hline & Usualmente no & 8 & 2,2 & 2,2 & 100,0 \\
\hline & Total & 361 & 100,0 & 100,0 & \\
\hline
\end{tabular}

Fuente: Estudiantes universitarios de la ULEAM - UPSE. Elaborado por: autores. 
La credibilidad de la actividad periodística siempre se encuentra en tela de juicio los datos recolectados en la encuesta indican que el $87 \%$ de los estudiantes indican que siempre y usualmente los medios de comunicación producen información falsa, con el objeto de manipular la opinión pública.

En la actualidad la labor del periodista se encuentra fiscalizada por la ciudadanía, ellos son los que valoran o avalan el desarrollo de esta actividad, particularmente en el contenido de la información que los medios presentan a la población, los ciudadanos de hoy gozan de diferentes instrumentos para enterarse de los hechos acontecidos y que la noticia puede ser contrastada y verificada por diferentes herramientas tecnológicas. Por lo tanto, se debe evitar cualquier tipo de situación que le reste profesionalismo al periodismo; como son las noticias falsas con o sin mala intención, los sesgos en los contenidos, que se puede constituir en una forma de falsear la verdad al estar su contenido sesgado, al respecto los estudiantes manifiestan que el $95 \%$ de usualmente presentan un sesgo en la información.

En este contexto, las tecnologías de información tienen un protagonismo, se propuso algunas preguntas para conocer la respuesta de los encuestados con relación a la credibilidad de la información recibida de las redes sociales. Particularmente en una doble dirección a saber; ¿Le concede credibilidad a la información que recibe por las redes sociales? Además de conocer; ¿La información recibida en las redes sociales favorece a la construcción de criterios para la opinión pública? Al respecto se recogieron los siguientes resultados:

Tabla 4. ¿Le concede credibilidad a la información que recibe por las redes sociales?

\begin{tabular}{|c|c|c|c|c|c|}
\hline & & Frecuencia & Porcentaje & Porcentaje válido & Porcentaje acumulado \\
\hline & Siempre & 11 & 3,0 & 3,0 & 3,0 \\
\hline & Usualmente & 107 & 29,6 & 29,6 & 32,7 \\
\hline & Ocasionalmente & 171 & 47,4 & 47,4 & 80,1 \\
\hline & Usualmente no & 66 & 18,3 & 18,3 & 98,3 \\
\hline & Nunca & 6 & 1,7 & 1,7 & 100,0 \\
\hline & Total & 361 & 100,0 & 100,0 & \\
\hline
\end{tabular}

Fuente: Estudiantes universitarios de la ULEAM - UPSE. Elaborado por: autores.

La respuesta de los encuestados a la siguiente pregunta es muy importante, puesto que indica que el $80,1 \%$ de la población le concede credibilidad a la información que reciben por las redes sociales, lo que representa y se convierte en un verdadero desafío, pero también una oportunidad para mantener la credibilidad de las mass medias. Con relación a la pregunta ¿La información recibida en las redes sociales favorece a la construcción de criterios para la opinión pública? En un porcentaje acumulado del 61,2\% indican que la información recibida favorece a la construcción de opinión pública. 
Tabla 7. ¿La contrastación de datos permite elaborar buenos contenidos noticiosos?

Frecuencia Porcentaje Porcentaje válido Porcentaje acumulado

\begin{tabular}{lrrrrr}
\hline Totalmente De Acuerdo & 161 & 44,6 & 44,6 & 44,6 \\
De Acuerdo & 162 & 44,9 & 44,9 & 89,5 \\
Válido & & & 8,6 & 8,6 & 98,1 \\
& Indeciso & 71 & 1,9 & 1,9 & 100,0 \\
\cline { 2 - 5 } & En Desacuerdo & 100,0 & 100,0 &
\end{tabular}

Fuente: Estudiantes universitarios de la ULEAM - UPSE. Elaborado por: autores.

La verificación de información, como parte del ejercicio periodístico puede pasar por un proceso de falibilismo, sin embargo, al ser la comunicación un proceso complejo y amplio, necesita ser contrastada y verificada con el fin de elaborar contenidos noticiosos lo más fielmente posible apegado a los hechos, es decir el factchecking, de esta manera se evitaría caer en la trampa de las Fake news, sin embargo esto se vuelve algo minúsculo frente la dimensión de las problemáticas que se plantean el diario vivir. Se puede inferir que los ciudadanos solicitan este tipo de tratamiento en la noticia el 89,5\% de los encuestados están de acuerdo con esta respuesta.

\section{Discusión}

En la perspectiva de la visión del consumidor de la información se identifica en este trabajo cómo el ciudadano construye o deconstruye la realidad, un tema que desde 1922 planteaba Wittgenstein.

Los resultados permiten interpretar datos trascendentales puesto que se lo puede contrastar con lo que indican los teóricos como Parrilla, quien manifiesta que la actividad periodística está bajo sospecha desde el siglo pasado, sin embargo, este tipo de situaciones se perciben con mayor ahínco en la actualidad, como lo manifiestan los encuestados. El probable que para evitar caer en esta situación es importante que los medios vuelvan chequear los datos como lo establecen sus preceptos y códigos deontológicos, además porque es un paso más dentro del proceso de rutinas productivas.

No obstante, el factchecking, visto y ejecutado desde el ciudadano promedio, probablemente, no sea algo que se haga con frecuenta, pues estarán quienes construyen desde su perspectiva, sin ejercer el falibilismo que es a lo que se refiere Popper. 


\section{Conclusiones}

Hacer una crítica desde la naturalización del ejercicio periodístico siempre será importante para la profesión, debido a que siempre existe la posibilidad de desviarse del propósito primigenio de la actividad.

La verificación de información, como parte del ejercicio periodístico puede pasar por un proceso de falibilismo, sin embargo, al ser la comunicación un proceso complejo y amplio que requiere de tiempo para investigar y constatar los datos.

La verificación de los contenidos periodísticos, a través de los mecanismos e instrumentos que proponen el factchecking, es indispensable para presentar un contenido acorde a los hechos. Por lo tanto, el chequeo de datos es un mecanismo que brinda la sensación de seguridad al consumidor de noticias.

Los resultados de la encuesta indican que la credibilidad de la actividad periodista está en tela de juicio. Por lo tanto, se debe evitar cualquier tipo de situación que le reste profesionalismo al periodismo, por lo que se necesita con urgencia un trabajo ético y con mucha responsabilidad.

Las redes sociales en la actualidad se están convirtiendo en generadoras de contenidos noticiosos y estos están construyendo opinión pública en la ciudadanía.

\section{Bibliografía}

Bachelard, G. (1938). La formación del espíritu científico. Buenos Aires: Siglo XXI, 12a edición, 1984.

Bauman, Z. (2015). Modernidad líquida. Fondo de cultura económica.

Eco Umberto. (1992). Interpretación y sobreinterpretación, Madrid: Cambridge University Press, $2^{a}$ edición, 1997.

González-Saibene, A. (2011). Conocimiento, intervención, transformación, Paraná: UNER.

Habermas J. (1968), Conocimiento e interés, Buenos Aires: Taurus, 1990.

Janowski, Kaz. (2013). Periodismo y comunicación científica, una buena dupla. Recuperado de: https://www.scidev.net/americalatina/comunicacion/editoriales/periodismo-y-comunicaci-n-cient-fica-unabuena-dupla.html

Luhmann, N. (2000): La realidad de los medios de masas. Barcelona: Anthropos. 
Parrilla. (2019). La imposibilidad del 'fact-checking', recuperado de: https://www.juandemariana.org/ijm-actualidad/analisis-diario/la-imposibilidaddel-fact-checking

Pascual, M. (2016). Política y propaganda: medios de comunicación y opinión pública. Editorial UOC.

Popper, K. (1998). Los dos problemas fundamentales de la epistemología. Madrid:

Tecnos.

Popper, K. y Candry, J. (1994): La Television: un danger pour la démocratie. Paris: Anatolie Editions.

Pujante, A. P. (2012). La lógica periodística en sentido epistemológico/The journalistic logic in epistemological sense. Estudios sobre el mensaje periodístico, 18(2), 891.

Schutz, A. (1973). Estudios sobre teoría social. Buenos Aires: Amorrortu.

Smiers, J. (2006). Un mundo sin copyright: artes y medios en la globalización (No. Sirsi) a454808).

Todorov, T. (1972) Presentación, en Lo verosímil, T. Contemporáneo, Bs As.

Tuchman, G. (1983). La producción de la noticia. Estudio sobre la construcción de la realidad. Barcelona, Gustavo Gili.

Tuchman, G. (1999). La objetividad como ritual estratégico: un análisis de las nociones de objetividad de los periodistas. CIC. Cuadernos de Información y Comunicación, $\mathrm{n}^{\mathbf{o}}$ 4, Madrid, Servicio de Publicaciones de la Universidad Complutense.

Vattimo, G. (1994). El pensamiento débil, Madrid: Cátedra, $3^{a}$ edición, 1995.

Verón, E. (2002). Construir el acontecimiento. Gedisa, Bs As.

Verón, E. (1972). Conducta, estructura y comunicación. Buenos Aires: Tiempo

Contemporáneo.

Wittgenstein L. (1922). Tractatus logico-philosophicus, Madrid: Alianza, 2007.

Watzlawick, P. (2011). ¿Es real la realidad?: confusión, desinformación, comunicación. Herder Editorial.

Zommer, L. (2014). El boom del fact checking en América Latina. Aprendizajes y desafíos del caso de Chequeado. Konrad adenauer stiftung. 